\title{
Fibre Optics Cabling Design for LHC Detectors Upgrade Using Variable Radiation Induced Attenuation Model
}

\author{
Mohammad Amin Shoaie \\ European Organization for Nuclear Research (CERN) \\ 1211 Geneva 23, Switzerland \\ E-mail: amin.shoaiedcern.ch \\ Jeremy Blanc \\ European Organization for Nuclear Research (CERN) \\ 1211 Geneva 23, Switzerland \\ E-mail: jeremy.blanc@cern. ch
}

\section{Simao Machado}

European Organization for Nuclear Research (CERN)

1211 Geneva 23, Switzerland

E-mail: simao.machado@cern.ch

\section{Daniel Ricci}

European Organization for Nuclear Research (CERN)

1211 Geneva 23, Switzerland

daniel.ricciecern.ch

Foreseen upgrades over the next decades enable LHC to operate at a higher luminosity (HL-LHC). Accordingly, the optical links designed to transmit particle collision data need to be hardened against increased radiation level, allowing for a reliable communication. In this paper we study the fibre cabling design of a link between the transceiver optical front-end and the data control room. The radiation penalty calculation takes temperature drop down to $-30^{\circ} \mathrm{C}$ into account. The proposed solution concatenates radiation-resistance and conventional fibres using multi-fibre interconnections. The end-to-end link loss during HL-LHC lifetime is estimated strictly less than $3.5 \mathrm{~dB}$ complying with predefined margin.

Topical Workshop on Electronics for Particle Physics

11 - 14 September 2017

Santa Cruz, California 


\section{Introduction}

High speed optical links are critical building blocks of LHC Experiments as they transmit particle collision data to the control room immediately after detection. In this respect CERN Versatile Link (VL) project has been active since 2008 to provide point-to-point bi-directional links for all LHC experiments [1]. The high radiation dose in proximity to these links necessitates special design criteria for active and passive components to ensure their performance during the accelerator run-time. With the foreseen upgrades in HL-LHC, the Versatile Link Plus (VL+) project was launched to upgrades the current links to reach 5-10 Gbps upstream and 2.5-5 Gbps downstream data rate and to provide the stringent design parameters for operating in a higher radiation dose [2]. The generic cable path for this link starts inside the detector volume (e.x CMS tracker as shown in Figure 1) and ends at the data control room.

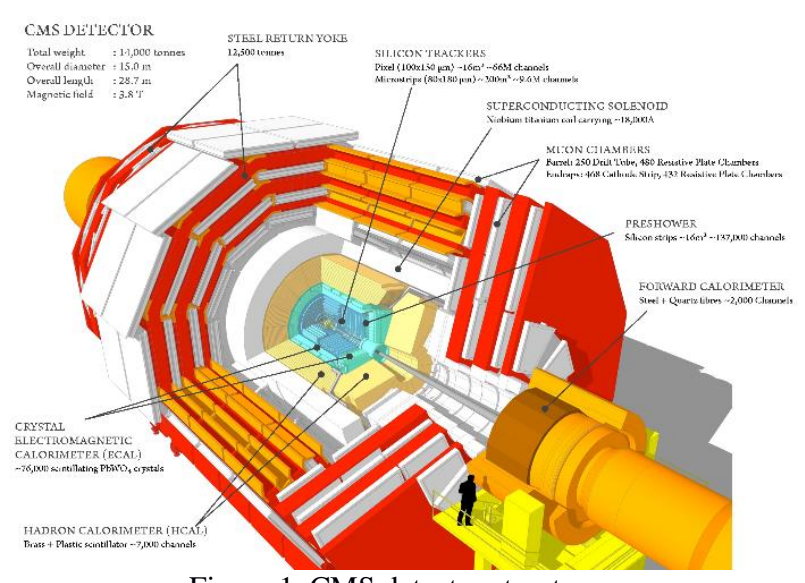

Figure 1. CMS detector structure.

\section{Investigation and Design}

\subsection{Radiation Test}

A critical design parameter of the link is to minimize the aggregated Radiation Induced Attenuation (RIA) which is a function of accumulated dose, irradiation dose-rate, fibre temperature and transmitted power over the fibre [3]. Figure 2 shows the simulated radiation map inside the ATLAS and CMS detector cross-section over a 10-year interval [4]. It can be verified that the radiation dose varies with the relative distance and radius from the beamline with maximum $10 \mathrm{MGy}$ in the close proximity of the beamline. The final cabling design should assure that the optical links are functional under this radiation condition.
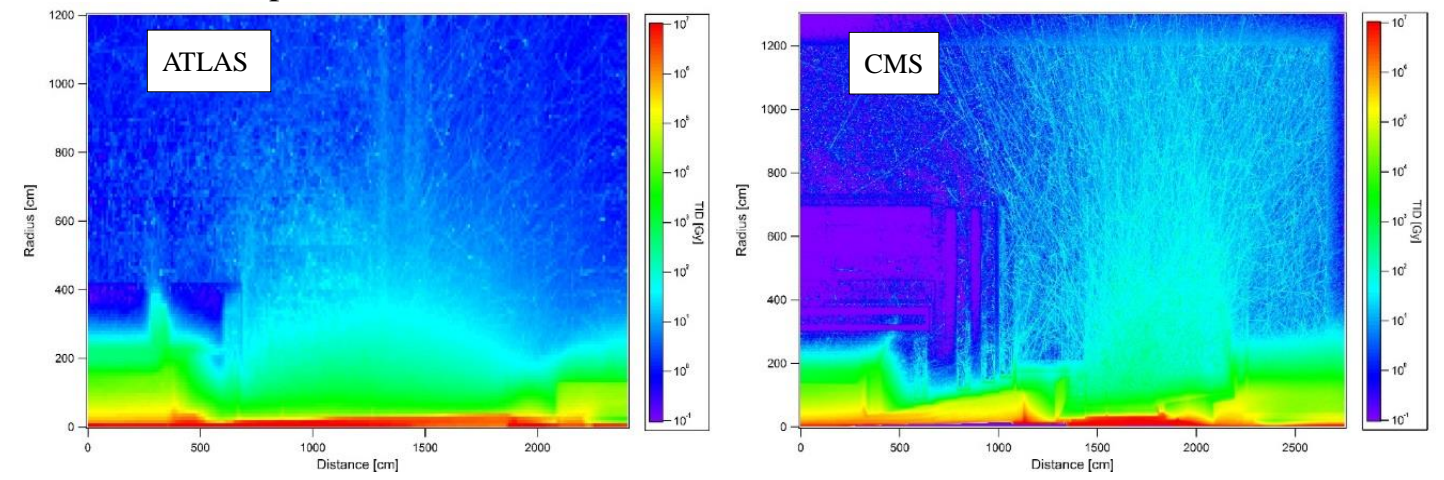

Figure 2. Simulated radiation map inside the ATLAS and CMS detector cross section over a 10-year interval [4]. 
Once the cable path is determined, the radiation penalty, $P$ is examined for three candidate Multi-Mode (MM) fibre types by integrating the incremental RIA element $d P=f(\Gamma(r), T(t)) \mathrm{d} r$ over the fibre length, $d r$. The $f$ function approximates piecewise RIA given the accumulated dose, $\Gamma$ and fibre temperature, $T$ as the arguments. We evaluated this function experimentally for tested fibres at accumulated dose $\Gamma$ up to $1 \mathrm{MGy}$ and the temperatures set $T=\{-30,25\}^{\circ} \mathrm{C}$ respectively corresponding to the inner and outer detector ambient temperature.

Figure 3 shows the experimental RIA results for 3 fibre types using Co-60 source at accumulated dose up to $1 \mathrm{MGy}$. As expected the RIA is enhanced in lower temperature [5]. The summarized results which are shown in Table 1, signify that a specific Radiation-Resistant (RR) fibre (Fibre A) outperformed other tested fibres (Fibre B and Fibre C) at both tested temperatures.

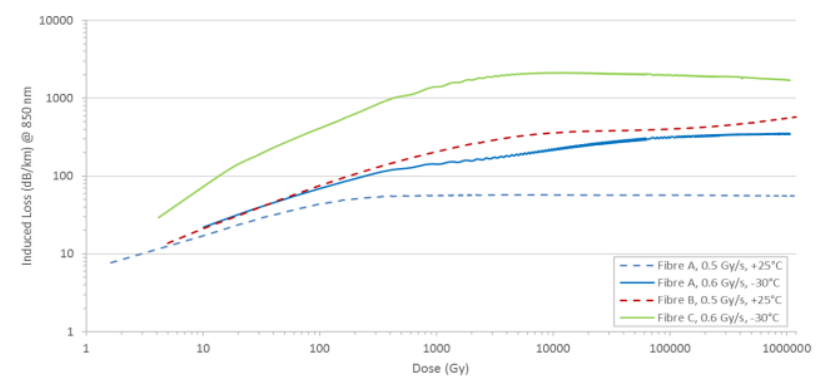

Figure 3. RIA versus accumulated dose up to 1 MGy for 3 fibre types.

Table1. Summarized RIA results at different dose and temperature for 3 fibre types.

\begin{tabular}{ccccc} 
Dose & $\begin{array}{c}\text { Fibre A } \\
\left(+25^{\circ} \mathrm{C}\right)\end{array}$ & $\begin{array}{c}\text { Fibre A } \\
\left(-30^{\circ} \mathrm{C}\right) \\
\mathrm{dB} / \mathrm{km}\end{array}$ & $\begin{array}{c}\text { Fibre B } \\
\left(+25^{\circ} \mathrm{C}\right)\end{array}$ & $\begin{array}{c}\text { Fibre C } \\
\left(\begin{array}{c}\left.-30^{\circ} \mathrm{C}\right) \\
\mathrm{dB} / \mathrm{km}\end{array}\right.\end{array}$ \\
\hline $1 \mathrm{kGy}$ & 56 & 142 & 205 & 1250 \\
\hline $10 \mathrm{kGy}$ & 57 & 220 & 360 & 2050 \\
$100 \mathrm{kGy}$ & 57 & 310 & 405 & 2000 \\
\hline $1 \mathrm{MGy}$ & 56 & 350 & 555 & 1500 \\
\hline
\end{tabular}

\subsection{Cable Path Design Example}

The cabling path is mainly determined by the available routing space inside the detector area as well as the arrangement of transceiver modules. The final path is designed to route the optical front-end inside the detector volume to the outer connection point through the existing cabling track. The path continues to the control room passing through the standard cable trays.

The fibre type used in the cables is selected so that the aggregated RIA is minimized while considering a cost efficient solution. Using the radiation map in Figure 2, two distinct radiation zones can be identified over the cable path with high and low RIA respectively inside and outside the detector area. Therefore as a cost efficient solution, the final design which is demonstrated in Figure 4 concatenates the RR fibre at the front-end fan-out and patch cord and a standard fibre at the trunk cable and back-end patch cord with interconnection inside the tracker and close to the detector solenoid. The interconnections are realized by multi-fibre (MPO) connectors. At the front-end side, 12-fibre MPO are used to adapt to the transceiver design, while 24-fibre MPO are employed at the back-end side to improve the cable space efficiency. 


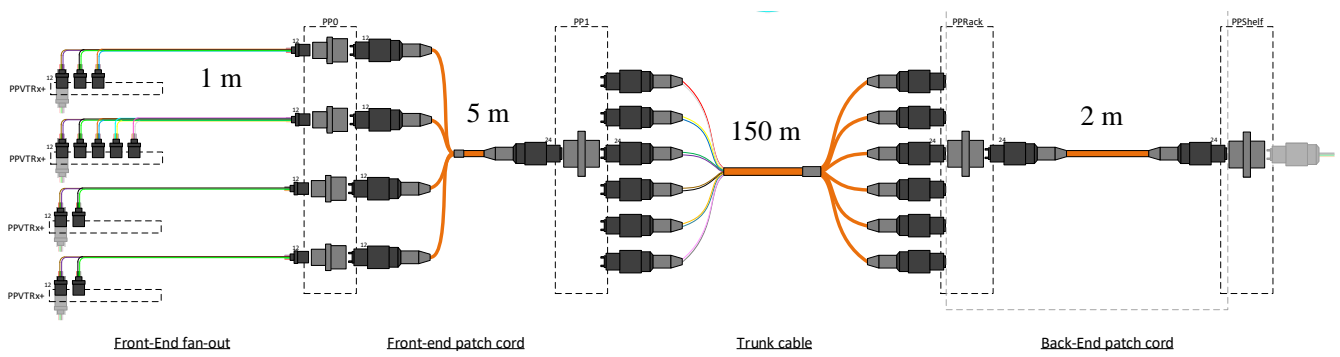

Figure 4. Cabling design concatenates a radiation resistant fibre at the front-end fan-out and patch cord and a standard fibre at the trunk cable and back-end patch cord sector.

\section{Results}

Given the existing guiding channels, the Total Ionizing Dose (TID) upper bound for the HL-LHC was calculated for CMS and ATLAS detectors in Figure 5 (a),(b) along the cable path. Comparing Figure 5 (a) and 5 (b) shows the predefined path for CMS is located closer to beamline axis and therefore is exposed to higher radiation level. Also CMS design employs two patching panels namely PP0 and PP1 whereas ATLAS design in based on a single patching panel. Using the RIA data in Figure 3 and TID in Figure 5 (a),(b) we obtained the accumulated RIA reversely along the cable path in Figure 5 (c),(d). It can be verified that the accumulated RIA is zero at the end of cable. However, as we move toward the beginning of the cable, it increases as a function of TID and fibre type. The accumulated RIA growth remains almost linear at high radiation levels due to low variation of RIA at that radiation level as shown in Figure 3. As mentioned before, the front-end side of links is made of RR fibre (Type A) to minimize the detrimental radiation effects. Standard fibre (Type B) is used for the remaining non-radiative areas. Therefore, the accumulated RIA during the 10 years interval was calculated $1.32 \mathrm{~dB}$ and $1.4 \mathrm{~dB}$, respectively for CMS and ATLAS cable path. Assuming maximum $0.38 \mathrm{~dB}$ propagation loss and $1.75 \mathrm{~dB}$ for five optical interconnections by 12 -fibre and 24-fibre MPO ferrules, the end-to-end link loss is in the order of $3.5 \mathrm{~dB}$ which is below the link margin set at $3.7 \mathrm{~dB}$. It worth noting that optical connectors using multi-fibre MT and MPO ferrules are shown to have negligible loss due to radiation in the presumed radiation levels [6].
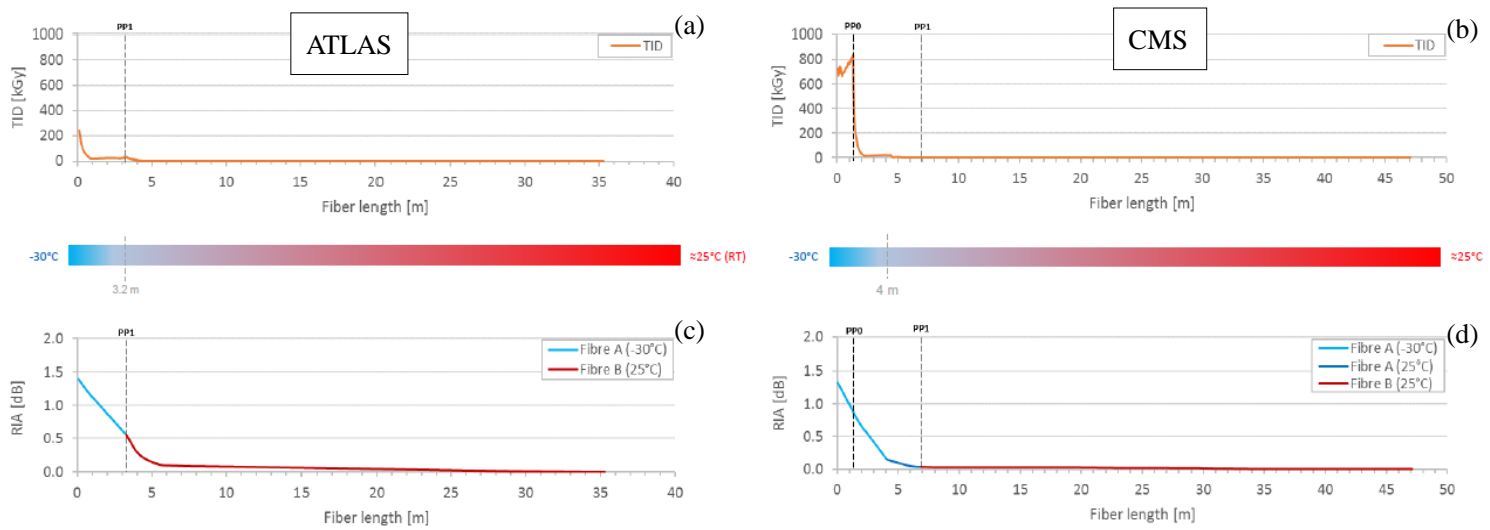

Figure 5. Total ionizing dose (TID) and radiation induced attenuation (RIA) for CMS and ATLAS cabling path design. 


\section{Conclusion}

A generic optical fibre cabling path was designed to provide the connectivity of optical transceiver modules inside the detector area to the data control room for CMS tracker. The design employs both radiation resistance and standard multi-mode fibres while the logic can be generalized to other detectors as well. The overall RIA together with propagation loss and interconnection loss is estimated $3.5 \mathrm{~dB}$ for the end-to-end link which is below the predefined margin set at $3.7 \mathrm{~dB}$.

\section{References}

[1] L. Amaral, S. Dris, A. Gerardin, T. Huffman, C. Issever, A.J. Pacheco, M. Jones, S. Kwan, S.C. Lee, Z. Liang, and T. Liu, 2009. The versatile link, a common project for super-LHC. Journal of Instrumentation, 4(12), p.P12003.

[2] Soós, C., Détraz, S., Olanterä, L., Sigaud, C., Troska, J., Vasey, F. and Zeiler, M., 2017. Versatile Link PLUS transceiver development. Journal of Instrumentation, 12(03), p.C03068.

[3] Hall, D., Huffman, B.T. and Weidberg, A., 2012. The radiation induced attenuation of optical fibres below$20^{\circ} \mathrm{C}$ exposed to lifetime $\mathrm{HL}-\mathrm{LHC}$ doses at a dose rate of $700 \mathrm{~Gy}(\mathrm{Si}) / \mathrm{hr}$. Journal of Instrumentation, 7(01), p.C01047.

[4] Schmidt, B. 2016 "The High-Luminosity upgrade of the LHC: Physics and Technology Challenges for the Accelerator and the Experiments" In Journal of Physics: Conference Series (Vol. 706, No. 2, p. 022002). IOP Publishing

[5] Blanc, J., Ricci, D., Kuhnhenn, J., Weinand, U., \& Schumann, O. J. (2017). Irradiation of Radiation-Tolerant Single-Mode Optical Fibers at Cryogenic Temperature. Journal of Lightwave Technology, 35(10), 1929-1935.

[6] Huffman, B. T., and A. Weidberg. "Summary of the effects of radiation upon the passive optical components of the Versatile Link." Journal of Instrumentation 9, no. 01 (2014) 